\title{
Correlação entre a composição corporal e a altura do salto em atletas profissionais de voleibol
}

\author{
Correlation between body composition and jump height in professional volleyball athletes
}

\author{
Vitor Xavier Crivelin ${ }^{1}$ \\ Alexandre Moreira ${ }^{2}$ \\ Ronaldo Luiz Finotti ${ }^{3}$ \\ Charles Ricardo Lopes ${ }^{4}$ \\ Mayara Ramos ${ }^{3}$ \\ Marcelo Saldanha Aoki ${ }^{3}$ \\ Caroline Dário Capitani ${ }^{1, *}$
}

\section{Resumo}

Objetivo: O presente estudo teve como objetivo descrever o comportamento dos parâmetros antropométricos de jogadores profissionais de voleibol ao longo do período competitivo e investigar a existência de correlação entre o perfil antropométrico e a altura do salto vertical nesses jogadores. Métodos: A composição corporal nos jogadores $(n=13)$ foi determinada por meio da mensuração de 7 dobras cutâneas, utilizando adipômetro. A altura do salto contra movimento foi feita por meio do tapete de contato em 3 tentativas, obtendo a média entre os resultados. A coleta dos dados foi realizada em 3 momentos da temporada competitiva (C1, C2, C3). Foi utilizado a ANOVA one-way e post hoc com Tukey. Posteriormente, foi determinado o coeficiente de correlação de Pearson. O nível de significância adotado foi de $p<0,05$. Resultados: Não houve diferença estatística em função do tempo para todas as medidas corporais e a altura do salto $(p>0.05)$. Foi observada correlação $(C 1$ : $r=0,75$; $C 2$ : $r=0,76$; $C 3$ : $r=0,75, p<0.01)$ entre a massa magra $(\mathrm{kg})$ e a altura do salto $(\mathrm{cm})$. Também, foi detectada correlação (C1: $r=0,92$; $C 2: r=0,92$; $C 3: r=0,93, p<0.001$ ) entre a massa magra $(\%)$ e a altura do salto $(\mathrm{cm})$. Conclusão: A massa magra apresentou correlação com a altura do salto em jogadores de voleibol profissional. Os resultados sugerem que a massa magra deve ser monitorada ao longo da temporada competitiva, considerando sua relação com o desempenho de saltos.
\end{abstract}

Palavras-chave: voleibol, perfil antropométrico, altura do salto, atletas, desempenho.

\begin{abstract}
Objectives: The present study aimed to describe the anthropometric profile of professional volleyball players during the competitive season and to investigate the relationship between the anthropometric profile and the jump height in these players. Methods: Body composition was assessed using a 7 skinfolds protocol, with a calibrated caliper. Counter-Movement Jump Height was measured with a jump mat in 3 attempts, and the mean value was retained for analysis. The data collection was conducted in 3 moments of the competitive season $(\mathrm{C} 1, \mathrm{C} 2, \mathrm{C} 3)$. Also, one-way ANOVA was used followed by the Tukey post hoc. In addition, the Pearson correlation coefficient was determined. The level of statistical significance was at $p<0,05$. Results: There was no statistical difference $(p<0,05)$ in body composition and jump height during the competitive season. A strong correlation (C1: $r=0,75 ; C 2: r=0,76 ; C 3: r=0,75$, $\mathrm{p}<0,01)$ between absolute free fat mass $(\mathrm{kg})$ and jump height $(\mathrm{cm})$. In addition, there was a strong correlation ( $C 1: r=0,92 ; C 2: r=0,92 ; C 3: r=0,93, p<0,001)$ between relative free fat mass $(\%)$ and jump height $(\mathrm{cm})$. Conclusion: Free fat mass was positively correlated with jump height $(\mathrm{cm})$ in professional volleyball players. The results suggest that free fat mass should be constantly monitored during the competitive season, considering its relationship with jump performance.
\end{abstract}

Key-words: volleyball, anthropometric profile, jump height, athletes, performance.
Afiliação dos autores

${ }^{1}$ Faculdade de Ciências Aplicadas, Universidade de Campinas, Limeira, São Paulo, Brasil.

${ }^{2}$ Escola de Educação Física e

Esporte, Universidade de São Paulo, São Paulo, São Paulo, Brasil.

${ }^{3}$ Escola de Artes, Ciências e

Humanidade da Universidade de São Paulo, São Paulo, São Paulo, Brasil.

${ }^{4}$ Faculdade de Educação Física, Universidade Metodista de Piracicaba, Piracicaba, São Paulo, Brasil.

\section{${ }^{*}$ Autor correspondente}

Universidade Estadual de Campinas, Faculdade de Ciências Aplicadas , R. Pedro Zaccaria, 1300 - Jd. Santa Luiza, Limeira, SP, Brasil.

e-mail:

caroline.capitani@fca.unicamp.br

\section{Conflito de interesses}

Os autores declararam não haver conflito de interesses.

\section{Processo de arbitragem}

Recebido: 14/09/2017 Aprovado: 19/04/2018 


\section{Introdução}

O voleibol é um esporte complexo, que exige o desenvolvimento de diversas capacidades e habilidades motoras, por parte dos seus praticantes. As características observadas nos jogadores são: o nível técnico, o perfil antropométrico e capacidades físicas ${ }^{1}$. Além disso, a especificidade das diferentes posições impõe diferentes demandas técnicas e fisiológicas ${ }^{2}$. Dentre as capacidades físicas necessárias para o sucesso na partida de voleibol, a altura do salto vertical é apontada como a característica mais importante ${ }^{1,3}$. Em uma partida, estima-se que a média de saltos realizados por atacantes fique em torno de 40 a 70 saltos $^{4,5}$, variando em função do volume de jogo. Ainda, as habilidades de ataque e bloqueio caracterizam aproximadamente $45 \%$ dos movimentos totais dos jogadores e cerca de $80 \%$ dos pontos de uma partida é dependente da realização do salto vertical ${ }^{6}$. A melhor performance do ataque e bloqueio está relacionada com a altura na qual os atletas conseguem atingir, pois existe correlação significante entre a altura do salto e as chances de sucesso de um bloqueio ou ataque ${ }^{7}$.

A composição corporal também parece ser capaz de afetar o desempenho dos atletas ${ }^{8}$. Atletas de alto nível possuem constituição corpórea diferente da população não-atleta. Os atletas de voleibol possuem maior quantidade de massa magra e também menor quantidade de gordura corporal $^{9}$, maior estatura e membros mais compridos ${ }^{10}$. Quanto à porcentagem de gordura corporal, não existe padrão de referência para o voleibol. Estudos prévios apresentam variação entre $11,2 \%$ e $22,3 \% \%^{7-9,14,15,20-23,27,30}$.

Entretanto, considerando a importância do desempenho de saltos para a modalidade, a gordura corporal poderia influenciar negativamente, impondo maior demanda energética e, consequentemente, causando exaustão precoce. Por outro lado, o aumento de massa magra poderia ser benéfico, pois contribui para o aumento de força ${ }^{12}$. Stamm et al. em $2003^{13}$ mostraram que as medidas antropométricas possuem influência relevante na performance de componentes técnicos e táticos, como o ataque e bloqueio. Ao avaliar as características antropométricas por posições, Mielgo-Ayuso et al. em $2015^{14}$ observaram que os líberos são os mais baixos e também os mais leves e que os jogadores mais altos são os menos ágeis. Esses autores também verificaram que a força dos membros superiores estava positivamente relacionada ao perímetro das panturrilhas ${ }^{14}$.

Além de ser considerado componente de aptidão física, o perfil antropométrico também pode ser um indicador de risco para lesões. Um estudo feito com jogadores de voleibol masculino e feminino mostrou que patologias associadas com o tendão patelar estão presentes em $41 \%$ dos jogadores ${ }^{15}$. Altura elevada, peso elevado e relação cintura/quadril acima do recomendado estão relacionados com o aparecimento de lesões no tendão patelar ${ }^{15}$. Atletas com circunferência do quadril maior ou igual a $83 \mathrm{~cm}$ possuem 2,5 vezes mais chances de desenvolver uma patologia no tendão patelar ${ }^{15}$. Dados antropométricos também estão relacionados com o nível de treinamento dos atletas. Estudo comparativo entre times nacionais, sub-19 e juniores, concluiu que os jogadores do time nacional possuem altura maior e apresentam maior alcance vertical em relação as duas outras divisões inferiores ${ }^{12}$

Diante do exposto, é razoável assumir que o acompanhamento das medidas antropométricas é relevante em modalidades como o voleibol, que dependem do desempenho de saltos verticais para a realização das principais ações de ataque e defesa. Dessa forma, o presente estudo teve como objetivo descrever o comportamento dos parâmetros antropométricos de jogadores profissionais de voleibol ao longo do período competitivo e ainda de investigar a existência de correlação entre o perfil antropométrico a altura do salto vertical em jogadores profissionais de voleibol.

\section{Métodos}

A abordagem experimental utilizada no presente estudo é do tipo transversal, observacional-descritiva, durante a primeira fase da SuperLiga 2015/2016. A amostra é constituída por atletas profissionais de voleibol, com idades entre 19 e 35 anos, pertencentes a equipe vice-campeã da SuperLiga. Os dados foram coletados durante o período de 2 meses (início; $\mathrm{C} 1,4{ }^{\text {a }}$ semana; C2 e $8^{\text {a }}$ semana; C3) referentes à primeira fase da
SuperLiga 2015/2016. O estudo teve início após aprovação do Comitê de ética em Pesquisa EEFE-USP (CAAE: 02313512.5.0000.5391) e assinatura do Termo de Consentimento Livre e Esclarecido (TCLE).

\section{Análise Corporal}

Foi realizado avaliação antropométrica individual com balança e estadiômetro, previamente, calibrados para determinação da massa corporal $(\mathrm{kg})$ e altura $(\mathrm{cm})$, respectivamente (Sanny, Brasil). A massa corporal fol mensurada para o $0,1 \mathrm{~kg}$ mais próximo e altura foi mensurada para $00,5 \mathrm{~cm}$ mais próximo. Aferiu-se as dobras cutâneas por meio do protocolo de 7 dobras $^{16}$ (peitoral, axilar, triciptal, subescapular, abdominal, supra-ilíaca e coxa), utilizando o adipômetro (Lange, EUA). Para estimar a Massa Magra (MM) $\mathrm{kg}$, Massa Magra Relativa (MMR) \%, Massa Gorda (MG) kg e Massa Gorda Relativa (MGR) \%, foi utilizada a equação bicompartimental $^{29}$, separando a composição corporal em MM e MG. O cálculo da estimativa de porcentagem corporal de gordura foi realizado por meio da equação: "Densidade Corporal $(\mathrm{DC})=1,11200000-[0,00043499$ (Somatória das Dobras) + $\left.0,00000055(\mathrm{SD})^{2}\right]-\left[0,0002882\right.$ (idade)] ${ }^{16}$ e após o resultado, DC é inserido em outra equação: "\%Gordura corporal = $[(4,95 / D C)-4,50] \times 100$ "29, sendo o valor final a porcentagem de gordura corporal do indivíduo. Foi realizado 3 medidas consecutivas para cada local, sendo a média retida para posterior análise. Durante o período de 2 meses, as coletas dos dados antropométricos foram realizadas 3 vezes, início (C1), $4^{\text {a }}$ semana (C2) e $8^{\mathrm{a}}$ semana (C3) da temporada competitiva.

\section{Análise do Impulso Vertical}

A avaliação da capacidade de impulsão vertical (altura do salto) foi realizada por meio do tapete de contato (CEFISE, Brasil), a partir da média de 3 repetições de saltos consecutivos, separados pelo intervalo de 5 minutos entre cada tentativa. A aplicação desse teste foi feita no início das sessões de treinamento, após o aquecimento, para que os atletas estivem em um estado ótimo de condição física. As coletas do salto vertical foram realizadas juntamente com análise antropométrica, ao longo de 2 meses, divididos em 3 encontros com a equipe. O padrão de movimentos utilizados nos testes de salto vertical foi realizado com contramovimento (SCM) e também com a utilização dos braços no auxílio do impulso ${ }^{17}$.

\section{Análise Estatística}

Os dados foram expressos em média \pm desvio padrão. A normalidade dos dados foi confirmada pelo teste de ShapiroWilk e para verificar se houve mudanças corporais em função do tempo, o método de análise ANOVA one-way e post hoc com teste de Tukey foi utilizado. Para investigar a correlação entre os dados antropométricos com as alturas atingidas na impulsão vertical, foi realizado o coeficiente de correlação de Pearson, com significância estabelecida em $p<0,05$. As análises foram conduzidas com o auxílio do software Office Excel 2013 e GraphPad 6.01.

\section{Resultados}

$\mathrm{Na}$ tabela 1 estão apresentados os valores obtidos pela análise antropométrica e também os valores da altura do salto sobre o tapete de contato, durante as 3 coletas. Não houve diferença estatística em função do tempo para todas as medidas corporais e também para a altura do salto $(p<0,05)$. Em geral, os parâmetros de composição corporal avaliados não oscilaram durante os 2 meses de coleta.

Os dados referentes à correlação entre as medidas antropométricas e as altura dos saltos estão apresentados na Tabela 2. Foi observada correlação significante $(r=0.717$; $\mathrm{p}<0,01)$ entre o peso $(\mathrm{kg})$ dos atletas e a altura do salto. Também foi detectada correlação significante (C1: $r=0,759$; $C 2$ : $r=0,762 ; \mathrm{C} 3: \mathrm{r}=0,755, \mathrm{p}<0.01)$ entre a MM $(\mathrm{kg})$ nas 3 coletas (C1, C2 e C3). Outro resultado encontrado foi a correlação significante $(C 1: r=0,928 ; C 2: r=0,928 ; C 3: r=0,931, p<0.001)$ entre a MMR (\%) e a altura dos saltos em todas as coletas (C1, C2 e C3). 
Tabela 1

Parâmetros antropométricos ao longo de 2 meses $(n=13)$. Valores apresentados em média \pm DP

\begin{tabular}{lccc}
\hline & $1^{\text {a }}$ Coleta & $2^{\text {a Coleta }}$ & $3^{\text {a }}$ Coleta \\
\hline Peso $(\mathrm{kg})$ & $90,6 \pm 11,2$ & $90,1 \pm 11,0$ & $91,0 \pm 11,6$ \\
Massa Magra (kg) & $81,6 \pm 10,9$ & $81,6 \pm 11,0$ & $81,9 \pm 11,2$ \\
Massa Gorda (kg) & $9,1 \pm 4,4$ & $8,5 \pm 4,2$ & $9,1 \pm 4,1$ \\
Massa Magra Relativa (\%) & $90,0 \pm 4,8$ & $90,6 \pm 4,8$ & $90,0 \pm 4,4$ \\
Massa Gorda Relativa (\%) & $10,0 \pm 4,8$ & $9,4 \pm 4,8$ & $10,0 \pm 4,4$ \\
Altura do salto (cm) & $54,4 \pm 7,0$ & $57,1 \pm 7,6$ & $51,8 \pm 5,0$ \\
\hline
\end{tabular}

Tabela 2

Correlação entre os parâmetros antropométricos e a altura do salto. Valores apresentados em média \pm DP.

\begin{tabular}{|c|c|c|c|}
\hline & \multicolumn{3}{|c|}{ Altura do salto $(\mathrm{cm})$} \\
\hline & $1^{\text {a }}$ Coleta & $2^{\text {a }}$ Coleta & $3^{\text {a }}$ Coleta \\
\hline Peso $(\mathrm{kg})$ & $0,717^{\star \star}$ & $0,724^{\star \star}$ & $0,758^{\star \star}$ \\
\hline Massa Magra (kg) & $0,759^{\star *}$ & $0,762^{\star \star \star}$ & $0,755^{\star \star}$ \\
\hline Massa Gorda $(\mathrm{kg})$ & $-0,062$ & $-0,062$ & 0,204 \\
\hline Massa Magra Relativa (\%) & $0,928^{\star \star \star}$ & $0,928^{\star \star \star}$ & $0,931^{\star \star \star}$ \\
\hline Massa Gorda Relativa (\%) & $-0,041$ & $-0,041$ & 0,238 \\
\hline
\end{tabular}

${ }^{\star \star} \mathrm{p}<0,001 ;{ }^{* \star *} \mathrm{p}<0,0001$

Em relação a esses dados, a quantidade de $M G(\mathrm{~kg})$ e a de MGR (\%) não apresentaram correlação com a altura dos saltos, demonstrando que, para esses atletas, a quantidade de gordura não se relaciona com a altura do salto.

\section{Discussão}

O presente estudo teve como objetivo descrever 0 comportamento dos parâmetros antropométricos de jogadores profissionais de voleibol ao longo do período competitivo e ainda de investigar a existência da correlação entre 0 perfil antropométrico e a altura do salto vertical em jogadores profissionais de voleibol.

Os principais resultados foram: 1) a não alteração dos parâmetros de composição corporal investigados em função do tempo (Peso total (kg), MM, MG, MMR, MGR) e altura do salto vertical $(\mathrm{cm}), 2)$ foi detectada correlação significante $(p<0,01)$ entre a MM para a altura do salto vertical $(\mathrm{cm})$ em todas as coletas e 3) foi observada correlação significante $(p<0,001)$ entre a MMR e a altura do salto (cm) nos 3 momentos, C1, C2 e C3.

Como dito anteriormente, não foi possível observar alteração significante na composição corporal e na altura do salto dos jogadores ao longo do tempo ( $\mathrm{C} 1, \mathrm{C} 2$ e $\mathrm{C} 3)$. Esses resultados podem ser explicados pelo período relativamente curto (2 meses) de observação desses parâmetros. Em trabalhos anteriores que demonstraram mudanças corporais significantes ao longo da temporada, foi utilizado períodos entre 4 meses e 2 $a^{3}$ s $^{3,8,21}$. Ainda, por se tratar de uma população altamente treinada, as respostas aos estímulos de treinamento desses indivíduos são menores, necessitando de uma combinação de estímulos diferenciados por longos períodos para que alguma adaptação seja perceptível. Uma observação interessante é que a manutenção desses parâmetros nos indica que os atletas não estão em déficit calórico em período competitivo, o que poderia ser negativo para o desempenho.

Além da composição corporal dos atletas, a altura do salto é determinante para atingir um resultado satisfatório nas partidas, sendo essa a característica mais importante para um jogador de voleibol $^{1,3}$. O presente estudo encontrou resultados de C1: 54,4 $\pm 7,0 \mathrm{~cm}$; C2: $57,1 \pm 7,6 \mathrm{~cm}$; C3: $51 \pm 5,0 \mathrm{~cm}$ para a altura dos saltos, obtendo a média entre os 3 períodos de $54,1 \pm 6,8 \mathrm{~cm}$, não separando os atletas por tamanho ou posição. Estudos anteriores encontraram resultados semelhantes em relação à impulsão vertical. Aouadi et al. em $2012^{23}$ encontrou valores de $50,0 \pm 3,9$ para o SCM com o balanço do braço. Sattler et al. em $2015^{25}$ identificou uma altura no SCM de 49,1 $\pm 5,6 \mathrm{~cm}$ para jogadores profissionais. Jogadores da liga regional italiana tiveram uma média de $47,9 \pm 5,7 \mathrm{~cm}^{26}$, jogadores da liga nacional belga obtiveram resultados bem semelhantes encontrados no presente estudo, $56,5 \pm 4,6 \mathrm{~cm}$ para atletas da primeira divisão e de $51,2 \pm 2,3 \mathrm{~cm}$ para atletas da segunda divisão ${ }^{27}$. Estudos realizados com a seleção espanhola de voleibol observou valores de $56,5 \pm 3,5 \mathrm{~cm}$ para o time de 2006 , $56,8 \pm 6,4 \mathrm{~cm}$ para o time de 2007 e de $59,8 \pm 5,1 \mathrm{~cm}$ para 0 time de $2008^{28}$. Esses valores comprovam que, apesar da regionalidade dos jogadores, as alturas dos saltos são similares quando comparados em mesmo nível de treinamento. Portanto, por meio da compreensão das características corporais e da altura do salto, foi levantada a hipótese de que existiria relação entre esses parâmetros, pois os saltos verticais são ações de jogo dependentes da condição atual do atleta, portanto, investigar a existência dessa relação é fundamental para o processo de preparação de atletas de voleibol.

Foi possível observar forte correlação entre o peso total dos atletas e a altura do salto. Os valores de $\mathrm{R}$ encontrados para os três momentos no estudo foram de $0,717,0,724,0,758$, respectivamente. No entanto, os mesmos resultados não foram encontrados em estudos anteriores, nos quais não foi observada relação entre esses parâmetros $\left(r=0,087^{14}\right.$ e $\left.r=0,170^{23}\right)$. O primeiro estudo ${ }^{14}$ foi realizado com atletas profissionais do sexo feminino, que apresentaram média da altura de salto vertical menor do que a do presente estudo. Ainda, o peso total dessas atletas apresentou grande variação, sendo a atleta com o menor peso de $52,6 \mathrm{~kg}$ e com maior peso de $79,1 \mathrm{~kg}$. A mensuração da composição corporal foi realizada por um método diferente do aplicado no presente estudo, logo a comparação entre os resultados requer cautela. Porém, nesse mesmo estudo, a força gerada durante o salto vertical demonstrou forte correlação com o peso total $(r=0,746)$. No segundo estudo, a correlação entre o peso total e a altura atingida durante o salto foi de $r=0,170^{23}$. Nesse estudo participaram atletas profissionais com média de peso total menor do que a encontrada no presente estudo, mas com valores de salto vertical próximos. A diferença dos resultados pode ser devido à grande heterogeneidade das amostras utilizadas nesses estudos ${ }^{14,23}$, enquanto que 0 atual trabalho utilizou uma população mais homogênea. Além disso, os resultados do presente estudo levam em consideração que os atletas mais pesados conseguem saltar mais, e isso porque esses atletas provavelmente são mais pesados (e provavelmente possuem mais $M M$ ), são mais altos $e$ provavelmente possuem membros inferiores mais longos. Já foi demonstrado que o tamanho dos membros inferiores está correlacionado $(r=0,69)$ com a melhor performance do salto ${ }^{7}$.

Existe ainda, a contribuição da massa muscular em produzir trabalho dentro da divisão do peso total. É possível que a correlação do peso total atinja um determinado limite, um platô, visto que a quantidade de MG e MGR possuem correlações desprezíveis e em alguns momentos até negativa de acordo com resultados do presente estudo. Os dados reforçam a ideia de que o peso total tem uma importante parcela na contribuição da altura atingida, visto que o peso total foi semelhante em três momentos distintos.

Em relação aos resultados da MM e MMR, a média desses parâmetros entre C1, C2 e C3 é de 81,2 $\pm 10,4(\mathrm{~kg})$ e de 90,2 \pm $4,5(\%)$ respectivamente. Esse resultado vai de encontro com resultado anterior que analisou indivíduos de times nacionais: $77,9 \pm 7,1(\mathrm{~kg})^{20}$. Para os valores de MMR, resultado semelhante foi encontrado: $87,7 \pm 1,9^{24}$. A maioria dos estudos destacam os resultados antropométricos do peso total $(\mathrm{kg})$ e da gordura corporal (\%), não especificando os valores relacionados a MM e MMR, o que dificulta as comparações. Ademais, esses dados apresentados corroboram os resultados do presente estudo.

Para as correlações realizadas com a $\mathrm{MM}$, os valores encontrados foram de $r=0,759 ; 0,762 ; 0,755$, indicando forte correlação entre a quantidade de $\mathrm{MM}$ e altura do salto vertical. Ainda mais surpreendente, foram os resultados encontrados entre a MMR e a altura do salto vertical $(r=0,928 ; 0,928 ; 0,931)$, apresentando correlações muito forte entre esses parâmetros. Como elucidado por outros estudos ${ }^{9,19}$, os atletas de voleibol possuem maior quantidade de MM e MMR e também menores quantidades de MG e MGR quando comparados com populações que não praticam atividade física ${ }^{10}$. Ainda, as correlações podem ser justificadas pelo fato de salto vertical ser de natureza explosiva, o que necessita de elevada potência muscular ${ }^{12}$

Em relação a MG dos atletas, especificamente no voleibol, por se tratar de um esporte com uma alta quantidade de saltos, a gordura corporal pode influenciar negativamente exigindo maior demanda energética e uma exaustão precoce. A correlação entre a quantidade de MG $(r=-0,062 ;-0,062 ; 0,204)$ e a MGR $(r=-0,041 ;-0,041 ; 0,238)$ nos atletas não foi significante, ou seja, para a população de jogadores profissionais em específico, não existe influência da porcentagem de gordura com a altura do salto, não sendo esse um bom parâmetro para predizer performance nesses atletas. Esse resultado deve ser interpretado com cautela, pois a população do presente trabalho tem baixíssima quantidade de gordura. Esses dados corroboram 
com as atuais recomendações do American College of Sports Medicine, onde não limitam a porcentagem de gordura corporal para os atletas de voleibol ${ }^{11}$.

Contudo, o estudo apresenta algumas limitações. A metodologia utilizada para mensuração da composição corporal é dependente da experiência e técnica utilizada pelo profissional com o adipômetro. Outro ponto que merece destaque é o reduzido tamanho da amostra.

\section{Conclusão}

A composição corporal é capaz de influenciar a altura do salto vertical de jogadores de voleibol. O peso total $(\mathrm{kg})$ e a quantidade de $M M(\mathrm{~kg})$ são fatores que influenciam na altura atingida no salto vertical. Mais especificamente, a quantidade relativa de $\mathrm{MM}(\%)$ apresentou ótima relação com a altura do salto vertical. Esses dados revelam a importância de monitorar a composição corporal dos atletas, pois esta possui impacto direto na performance de saltos, que é crucial para a execução de outras ações de ataque e defesa determinantes para o resultado do jogo.

\section{Agradecimentos}

Os autores agradecem a Comissão Técnica, em especial ao Prof. Alexandre Stanzioni, pelo apoio para realização dessa investigação.

\section{Referências}

1. Lidor R, Ziv G. Physical and Physiological Attributes of Female Volleyball Players-A Review. J Strength Cond Res [Internet]. 2010 Jul;24(7):1963-73. Available from: $\mathrm{n}=00124278-201007000-00037$

2. Duncan MJ. Anthropometric and physiological characteristics of junior elite $\begin{aligned} & \text { volleyball players * Commentary. Br J Sports Med [Internet]. } 2006 \text { May } \\ & \text { 10;40(7):649-51. }\end{aligned}$ Available
from: 10;40(7):649-51. Available from:
http://www.pubmedcentral.nih.gov/articlerender.fcgi?artid=2564319\&tool=p mcentrez\&rendertype $=$ abstrac

3. Marques MC, Tillaar R Van Den, Vescovi JD, González-Badillo JJ. Changes in strength and power performance in elite senior female professional volleyball players during the in-season: a case study. $J$ Strength Cond Res. 2008:22(4):1147-55.

4. Tillman MD, Hass CJ, Brunt D, Bennett GR. Jumping and Landing Techniques in Elite Women's Volleyball. J Sports Sci Med [Internet]. 2004 Mar;3(1):30-6. Available http://www.ncbi.nlm.nih.gov/pubmed/24497818

5. Polglaze $\mathrm{T}$. The physiological requirements of the positions in state league volleyball. Sport Coach [Internet]. 1992;(March). Available from: http://www.researchgate.net/publication/266850557

6. Voigt $\mathrm{H}$, Vetter $\mathrm{K}$. The value of strength-diagnostic for the structure of jump training in volleyball. Eur J Sport Sci [Internet]. 2003 Jun;3(3):1-10. Available

http://www.tandfonline.com/doi/abs/10.1080/17461390300073310

7. Fattahi A, Ameli M, Sadeghi H, Mahmoodi B. Relationship between anthropometric parameters with vertical jump in male elite volleyball players due to game's position. J Hum Sport Exerc [Internet] players due to game's position.
2012;7(3):714-26. 2012;7(3):714-26. Avail
http://rua.ua.es/dspace/handle/10045/24526

8. González-Ravé JM, Arija A, Clemente-Suarez V. Seasonal Changes in Jump Performance and Body Composition in Women Volleyball Players. J Strength Cond Res [Internet]. 2011 Jun;25(6):1492-501. Available from: http://www.ncbi.nlm.nih.gov/entrez/query.fcgi?cmd=Retrieve\&db=PubMed\& dopt $=$ Citation\&list uids $=21273911$

9. Zapolska J, Witczak K, Manczuk A, Ostrowska L, Mańczuk A, Ostrowska L Assessment of nutrition, supplementation and body composition parameters on the example of professional volleyball players. Rocz Państwowego Zakładu Hig [Internet]. 2014;65(3):235-42. Available from: http://www.ncbi.nlm.nih.gov/pubmed/25247804

10. Gabbett T, Georgieff B. Physiological and anthropometric characteristics of Australian junior national, state, and novice volleyball players. J Strength Cond Res [Internet]. 2007;21(3):902-8. Available from: http://www.ncbi.nlm.nih.gov/pubmed/17685708

11. Rodriguez NNR, Di Marco N, Langley S, DiMarco NM. American College of Sports Medicine, American Dietetic Association, and Dietitians of Canada joint position statement: Nutrition and athletic performance. Med Sci Sports Exerc. 2009;41(3):709-31.
12. Mal L. the Profile and Comparison of Body Composition of Elite Female Volleyball Players. 2010;42:90-7.

13. Stamm R, Veldre G, Stamm M, Thomson K, Kaarma H, Loko J, et al. Dependence of young female volleyballers' performance on their body build, physical abilities, and psycho-physiological properties. J Sports Med Phys Fitness [Internet]. 2003 Sep;43(3):291-9. Available from: http://www.ncbi.nlm.nih.gov/pubmed/14625509

14. Mielgo-Ayuso J, Calleja-González J, Clemente-Suárez VJ, Zourdos MC Influence of anthropometric profile on physical performance in elite female volleyballers in relation to playing position. Nutr Hosp [Internet]. 2014 Oct 6;31(2):849-57. Available http://www ncbinlm nih gov/pubmed/25617573

15. Malliaras P, Cook JL, Kent PM. Anthropometric risk factors for patellar tendon injury among volleyball players. $\mathrm{Br} J$ Sports Med [Internet]. 2007;41(4):259-63; discussion 263. Available from: http://www.pubmedcentral.nih.gov/articlerender.fcgi?artid=2658960\&tool=p mcentrez\&rendertype $=$ abstract

16. Jackson AS, Pollock ML. Generalized equations for predicting body density of men. $\mathrm{Br} J$ Nutr [Internet]. 1978 Nov 9:40(03):497. Available from: http://www.journals.cambridge.org/abstract S0007114578000689

17. Sattler T, Sekulic D, Hadzic V, Uljevic O, Dervisevic E. Vertical jumping tests in volleyball: reliability, validity and playing-position specifics. Strength Cond Res [Internet]. 2011 Sep;1. Available from: http://content.wkhealth.com/linkback/openurl?sid=WKPTLP:landingpage\&a $\mathrm{n}=00124278-900000000-98721$

18. Ugrinowitsch C, Barbanti VJ, Gonçalves A, Peres BA. Capacidade dos testes isocinéticos em predizer a "performance" no salto vertical em jogadores de voleibol. Rev Paul Educ Física. 2000;14(2):172-83.

19. Malá L, Malý T, Záhalka F, Bunc V. The profile and comparison of body composition of elite female volleyball players. Kinesiology [Internet]. Fakultet za Fizicku Kulturu; 2010 Jun;42(1):90-7. Available from: http://ezproxy.lib.ucf.edu/login?URL=http://search.ebscohost.com/login.asp $x$ ?direct=true \&db=psyh\&AN=2010-14805-010\&site=ehostlivelnlucilali@yahoo.de

20. Zaccagni L, Onisto N, Gualdi-Russo E. Biological characteristics and ageing in former elite volleyball players. J Sci Med Sport. 2009;12(6):66772 .

21. Sheppard JM, Ewton ROUN. LONG-TERM TRAINING ADAPTATIONS IN ELITE MALE VOLLEYBALL PLAYERS. J Strength Cond Res. 2012;26(8):2180-4.

22. Marques MC, Tillaar R van den, Gabbett TJ, Reis VM, González-Badillo JJ. Physical Fitness Qualities of Professional Volleyball Players: Determination of Positional Differences. J Strength Cond Res [Internet]. 2009 Jul;23(4):1106-11. Available from: http://content.wkhealth.com/linkback/openurl?sid=WKPTLP:landingpage\&a $n=00124278-200907000-00008$

23. Aouadi R, Jlid MC, Khalifa R, Hermassi S, Chelly MS, Van Den Tillaar R, et al. Association of anthropometric qualities with vertical jump performance in elite male volleyball players. J Sports Med Phys Fitness [Internet]. 2012 http://www.ncbi.nlm.nih.gov/pubmed/22327081

24. Laffaye G, Choukou MA. Gender Bias in the Effect of Dropping Height on Jumping Performance in Volleyball Players. J Strength Cond Res [Internet]. 2010 Aug;24(8):2143-8. Available from http://content.wkhealth.com/linkback/openurl?sid=WKPTLP:landingpage\&a $\mathrm{n}=00124278-201008000-00024$

25. Sattler T, Hadžić V, Dervišević E, Markovic G. Vertical jump performance of professional male and female volleyball players: effects of playing position and competition level. J Strength Cond Res [Internet]. 2015 Jun;29(6):1486-93. Available from: http://content.wkhealth.com/linkback/openurl?sid=WKPTLP:landingpage\&a $n=00126334-201308010-00002$

26. Maffiuletti NA, Dugnani S, Folz M, Di Pierno E, Mauro F. Effect of combined electrostimulation and plyometric training on vertical jump height. Med Sci Sports Exerc [Internet]. 2002 Oct;34(10):1638-44. Available from: http://www.ncbi.nlm.nih.gov/pubmed/12370566

27. Forthomme B. Factors Correlated With Volleyball Spike Velocity. Am J Sports Med [Internet]. 2005 Oct 1;33(10):1513-9. Available from: http://journal.ajsm.org/cgi/doi/10.1177/0363546505274935

28. Borràs $X$, Balius $X$, Drobnic $F$, Galilea $P$. Vertical jump assessment on volleyball: a follow-up of three seasons of a high-level volleyball team. $J$ strength Cond Res [Internet]. 2011 Jun;25(6):1686-94. Available from: http://www.ncbi.nlm.nih.gov/pubmed/21386730

29. Siri, W. E. 1961. Body composition from fluid spaces and density: Analysis of methods. Pp. 223-244 in Techniques for Measuring Body Composition, J. Brozek, editor; and A. Henschel, editor., eds. Washington, D.C. National Academy of Sciences.

30. Sheppard JM, Cronin JB, Gabbett TJ, McGuigan MR, Etxebarria N, Newton RU. Relative Importance of Strength, Power, and Anthropometric Measures to Jump Performance of Elite Volleyball Players. J Strength Cond Res [Internet]. 2008 May;22(3):758-65. Available from: http://content.wkhealth.com/linkback/openurl?sid=WKPTLP:landingpage\&a $\mathrm{n}=00124278-200805000-00016$ 